\title{
Understanding and Readiness in Facing IR 4.0 Future Skills Transformation among UPM Trainee Counsellors
}

\author{
Subash Balan ${ }^{1}$, Zaida Nor Zainudin ${ }^{1}$, Habibah Ab Jalil ${ }^{1}$ \\ ${ }^{1}$ Faculty of Educational Studies, Universiti Putra Malaysia, Serdang, Selangor, Malaysia \\ Correspondence: Zaida Nor Zainudin, Faculty of Educational Studies, Universiti Putra Malaysia, 43400 UPM \\ Serdang, Selangor, Malaysia. Tel: 603-9769-8210. E-mail: zaidanor@upm.edu.my
}

Received: September 3, 2021

Accepted: September 28, $2021 \quad$ Online Published: October 14, 2021

doi:10.5539/ass.v17n11p69

URL: https://doi.org/10.5539/ass.v17n11p69

\begin{abstract}
Industrial Revolution (IR) 4.0 refers to the integration of technology within a particular industry, which encompasses big data, data analytics, cloud computing, robots, artificial intelligence, as well as Internet of Things (IoT) technologies. This study aims to assess the understanding of trainee counsellors of IR4.0 future skills, and to investigate how trainee counsellors improve their overall understanding of IR4.0 and its readiness. The qualitative research design employed in this study involved semi-structured interviews. Four trainee counsellors were chosen through convenient sampling and interviewed in 15-20 minute sessions. The findings indicate that trainee counsellors have a strong grasp of IR4.0. The respondents acknowledged, however, that their comprehension of IR4.0 in connection with future counselling professions is only modest, owing to the institution's lack of formal educational exposure. Consequently, the respondents' readiness to face IR4.0 is dangerously low, with the majority expressing worry towards their adaptability in future career development. Finally, the study concluded that educational institutions are vital in teaching and equipping students to confront the global challenges presented by IR4.0. This study is important because it aids researchers to analyse information on the understanding, readiness, and effect of IR4.0 on future skills among trainee counsellors. Additionally, it helps educational institutions in recognising the essential role of IR4.0 adoption in teaching and learning, as well as the implementation of the necessary measures to increase the readiness for training counsellors in tackling IR4.0.
\end{abstract}

Keywords: Industrial Revolution 4.0, trainee counselors, undergraduates, future skills transformation, future skills knowledge, career transformation

\section{Introduction}

Industrial Revolution (IR) 4.0, announced by Germany in 2011, was envisioned as automation and data exchange in manufacturing technologies, facilitated primarily by multidisciplinary engineering facilitations that coordinate into a seamless connection. IR 4.0 is crucial in integrating and combining intelligent devices, human actors, and processes across organisational stages to create technological data-driven, systematic, and agile value chains. This may be accomplished by utilising technologies such as cyber-physical systems, the Internet of Things (IOI), big data and analytics, augmented reality, cognitive computing, and smart factory (Tay et al., 2018).

The Malaysian economy is steadily transitioning from labour-intensive to capital- and technology-intensive, which considerably enhances the relevance of the development of human resource policy, especially in the education and training sectors. To tackle the challenges of IR4.0, all students enrolling in Higher Education Institutions (HEI) must transcend their comfort zones and prepare for this new age, especially trainee counsellors. The quality of educators, including lecturers, instructors and counsellors, are crucial to equip future generations in tackling IR4.0. Educators are expected to have the experience and capacity to adapt and adopt the technological developments connected with IR4.0. Conventional literacy linked with reading, writing, science, and mathematics must be complemented with new literacy, including data literacy, technology, and human resources (Johari et al., 2021; Lase, 2019).

According to Abdullah et al. (2020), the recent topic of unemployment in Malaysia is mostly due to a lack of IR 4.0 skills among graduates of (HEI), which leads to unemployment. As a result, more efforts should be made to create graduate work competency preparedness of skill needed in line with IR 4.0 problems, ensuring that graduates are competent with IR 4.0 employment demand. In this context, trainee counsellors play significant 
roles, as they are soon-to-be teachers and counsellors in schools or colleges, where they will be instrumental in shaping the future abilities of school students and college students. The success of these aims relates to bringing creative and helpful counsellors to schools who can enable the students to learn new knowledge and motivate them to accept the changes of IR4.0.

Therefore, it becomes important to explore the understanding and readiness of trainee counsellors in facing IR4.0, in accordance with the technical developments that are the advent of IR4.0. This research explores participants' opinions towards their readiness and knowledge regarding future competencies. The study expects that the participants would draw comparisons of the present career transition with their prior expertise. This research is of specific relevance to the Department of Guidance and Counselling $(\mathrm{G} \& \mathrm{C})$ in the domain of education as it indicates that higher-level counsellors with high comprehension of IR4.0 may provide a better teaching and learning process to future generation trainee counsellors.

\subsection{Current Study}

The expansion and influence of IR4.0 have swiftly transformed conventional infrastructure-people systems into modernised systems that demand a professional workforce with cross-functional capabilities and advanced competences to manage new integrated processes and information technology (IT) systems (Sima et al., 2020). Thus, the primary problem is to ensure that new personnel entering the IR4.0 era have all the required automation, digital, and information technology skills, as well as the soft skills essential to manage and utilise the requirements of smart systems.

Educational competence, technology commercialisation skills, globalisation capacity, future strategy expertise, and counselling competence are the qualities that must be acquired by the educator. The national education system's major agenda is to produce educators and counsellors with high competence, knowledge, and abilities in order to contribute to effective learning among school students. According to Sima et al. (2020), the adoption of new technology has an impact on both workers and companies. To contribute to the realisation of IR4.0, it is essential that the educational system is adjusted to meet the new development needs of society. The primary human capital issues in IR4.0 are on adapting talents and human activities to diverse industrial needs as a result of process divergence. As a result, human capital plays a major role in a working environment and are prone to employment and educational redirection.

In today's rapidly changing technological world, the IR4.0 has emphasised the need to redesign the educational system, with a particular emphasis on transforming global learning and teaching methods. According to Abdul Kadira, et al. (2020), students' knowledge and preparation for IR4.0 are at an alarming level. Students, particularly trainee counsellors, should be prepared to execute and manage IR4.0's digital integration. This is critical to develop future workforces that are competent, knowledgeable, and skilled. Thus, the following research questions helps to accomplish the objectives:

$\mathrm{RQ}_{1}$ : What is the trainee counsellors' understanding of IR4.0 future skills?

$\mathrm{RQ}_{2}$ : How do trainee counsellors develop their understanding of IR4.0 and its readiness?

\subsection{Literature Review}

Artificial intelligence, machine learning, and automated technologies, along with data science, are used to govern a network of interconnected systems in IR4.0. In today's era of globalisation and digitalisation, formal education has become the key standard in the recruiting process. Career readiness comprises a set of abilities linked to success, comprehension, and personal attitude that must be acquired by counseling graduates to potentially get and keep the desired employment. Career readiness is closely connected with the capacity of counseling graduates to apply the abilities gained at the university and turn them into a meaningful contribution at a job. Universities and colleges are mainly responsible for training students for future jobs (Supian et al., 2020). However, the primary issue that impacts the job preparedness among counseling graduates in Malaysia is the absence of soft skills, which may be described as skills and qualities that represent the personality and attitudes of an individual.

Additionally, the graduates are typically faced with a skills gap issue when it comes to IR4.0 development. In Malaysia, there is a difference in opinion about the readiness of students or graduates to join the workforce. Employers and fresh graduates have divergent views on the main employability skills that the industry urgently needs. Graduates have said that they are prepared to join the labour market, but the employers reject this notion. Additionally, the industry argues that student interns lack critical thinking, oral communication, active listening, creativity, and innovation abilities (Supian et al., 2020).

IR4.0 has transformed the characteristics of a traditional workplace, and graduates are now required to acquire 
skills that are relevant to these industrial demands. Therefore, students must be equipped and taught to enhance their readiness to confront IR4.0 and its demands. It is also important to modify and enhance the education in order to match with the demands of industrial sectors within the context of IR4.0. To fulfil these ideals, educators, especially those in higher learning institutions, play a vital role in training and producing high quality graduates and increase graduates' understanding and readiness by enhancing the teaching and learning (Abdullah et al., 2020).

Hence, the trainee counsellors at UPM have to be ready and have a complete grasp of IR4.0 before joining the working sectors. The trainee counsellors must establish a firm basis to face the increasingly uncertain job environment. If more persons are being retrenched due to the lack of skills, and students do not acquire the needed competence linked to IR4.0, the concern on how graduates would be able to join the labour market later would arise (Manonmani et al., 2020).

Pedagogy or teaching technique is continuously changing because of the changing learning environment and technology. The important aspects of IR4.0 such as internet of things (IOT), big data, artificial intelligence, and cognitive computing should be critically employed in the teaching and learning process. Lai et al. (2020) argued that knowledge, attitude, and the abilities of educators are essential in the application of IR4.0 in the teaching and learning process. This generates a requirement for education sectors to equip future educators with thorough awareness of IR4.0 and their importance in the industry.

The understanding and readiness for IR4.0 is the key determinant of future transformation skills among trainee counsellors. Understanding IR4.0 allows these future counsellors to offer superior counselling services to future generations and incorporate technology into the administration of counselling. IR4.0 is mostly used as a teaching tool in education and learning. As a result, the knowledge of IR4.0 alone is seldom adequate to prepare trainee counsellors with future transformation capabilities. This understanding must be supported by both the readiness to confront the IR4.0 challenges, as well as the technical capabilities necessary to implement IR4.0 technology (Zulnaidi \& Majid, 2020).

According to Halili et al. (2021), IR4.0 in education entails a paradigm shift away from conventional passive learning pedagogies towards a more advanced and contemporary model that includes more advanced and modern digital technology to improve customised learning. Trainee counsellors are required to be prepared to adapt to a changing environment, ready to utilise their current talents, and rapidly acquire new ones in order to participate fully in society. As a result, higher education institutions must review and assess the extent to which their existing programmes offer training and real-world insights to graduates entering the workforce, as well as the steps they need to take to expand beyond theoretical and academic teaching ( $\mathrm{Pu}$ et al., 2021; Tay et al., 2018).

Research has been performed to measure the degree of readiness and understanding among trainee counsellors of University Putra Malaysia (UPM) in facing IR4.0. The trainee counsellors are the major focus in this research as they are the future counsellors that play key roles in the implementation of IR4.0 in future generations.

\section{Methodology}

In order to determine the optimum research design for this study, a qualitative research approach was used. Descriptive and narrative study data was deemed the best way to elicit relevant findings to the research questions, based on the research objective and questions (Merriam, 1998). Semi-structured interviews were utilised to gather data because they enable the respondents to express themselves in their own words. Online face-to-face interviews were employed to collect the qualitative data.

The focus of this research was on the students attached to the UPM, Faculty of Education, Guidance and Counselling (G\&C) Study department. Third-year undergraduates were chosen for this study. The entire population of undergraduates in this school is around 25 individuals in the third-year (final year) Bachelor of Education and Counselling (Guidance and Counselling) class. However, convenient sampling was then employed in order to select the undergraduates to be interviewed. Upon reaching a saturation threshold, a total of 4 undergraduates were interviewed, with each session 25-30 minutes in duration. The major aim of the interview session is to comprehend the respondent's viewpoint, ideas, and experiences on the topics being raised.

\subsection{Instrument}

In qualitative research, the person of the researcher is critical for the quality of the scientific knowledge and for the soundness of ethical decisions in any research project. By conducting interviews, the importance of the researcher as a person is magnified because the interviewer himself is the main instrument for obtaining knowledge.

Furthermore, the interview protocol is important to the researcher to increase the quality of the interview 
questions necessary for the study. In this study, the researcher sent the interview questions to an expert to acquire feedback on the interview protocol and enhance the reliability, anticipate the respondents' answers, and ensure understandability.

They are experts in the field of counselling and qualitative research. After receiving the feedback on the interview protocol, the researcher made the appropriate amendments. The semi-structured interview was guided by an interview protocol as listed below:

1. What is your understanding of IR4.0 future skills?

2. What are the IR4.0 future skills needed in a counselling job, practice, and career?

3. How do you develop understanding towards IR4.0?

4. How can IR4.0 readiness be enhanced among trainee counsellors?

\subsection{Sample}

The participants in this study were recruited through convenient sampling, or more specifically, a call for research volunteers. The announcement contained details about the current study, as well as how volunteers would be involved in data collection. Most importantly, those who volunteered were required to confirm that they are third-year students from the Counselling Department in the UPM Education Faculty. Separate online interviews were also conducted. Throughout the 25- to 30-minute long interviews, the interview protocol was continuously used as a reference.

The following table depicts the profile of the four participants involved in the present study. It is important to note that all the participants derive from the same class and faculty.

Table 1. Interview participants' profile follow

\begin{tabular}{cccc}
\hline \multirow{2}{*}{ Item } & Details & University & Total \\
\hline \multirow{2}{*}{ Gender } & Male & UPM & 2 \\
& Female & UPM & 2 \\
\hline
\end{tabular}

\section{Results and Discussion}

By analysing the interview data, the research questions were addressed and clarified. The study topics address the trainee counsellors' understanding of IR4.0 future skills and the process through which they gain understanding and readiness for IR4.0.

\subsection{Interview Sections}

The interview is divided into four distinct sections. The first section assesses the respondents' understanding of IR4.0 and its relationship with future skills, whereas the second section assesses the respondents' understanding of the relationship between IR4.0 and future career skills. The third section examines how respondents acquire and increase their understanding of IR4.0, and the final section assesses the trainee counsellors' readiness to face IR4.0.

\subsubsection{Understanding of IR4.0 Future Skills}

Trainee counsellors must be well-versed in and understand IR4.0 in order to offer accurate information and assist prospective students in establishing an interest in the subject. With a solid understanding of IR4.0, trained counsellors will be able to assist students in developing their own interests and talents that will in turn aid them in making professional decisions. Thus, the respondents were asked about their knowledge of IR4.0 future skills. The responses have shown a good grasp of the relation between IR4.0, digital literacy, and the usage of technology such as data analytics, artificial intelligence, and cloud computing. According to respondents, IR4.0 is associated with digital automation, especially in the industrial sector. Four of the respondents: R1, R2, R3, and R4 alluded to this notion:

"IR4.0 is concerned with technological advances such as the use of technology into pedagogy, teaching, and learning ..." (R1)

"IR4.0 entails the use of technology, particularly big data and digitisation in solving problems, especially in industry ..." (R2)

"IR4.0 is involved with digital literacy, which includes the usage of software programmes, data analytics, and technical gadgets ..." (R3) 
"IR4.0 is emphasis on the fields of STEM education, and the learning system is reoriented based on ICT learning ..." (R4)

Additionally, the respondents connect IR4.0 to a reduction in dependence on human labour and an increase in digital integration, which requires the use of IR4.0 technology such as robotics and artificial intelligence. The respondents are also able to correlate the implementation of IR4.0 to future jobs and have given specific instances. By incorporating IR4.0 into the curriculum, the educational system may focus on developing holistic, creative, and critical graduates. Implementing IR4.0 via coursework and job-based learning will also improve educational quality and enhance the undergraduates' understanding of IR4.0. This study indicates that the respondents have demonstrated a broad knowledge of IR4.0 and its implications for future careers.

However, the results of this study contradict those of Ismail et al. (2020), who discovered that students' understanding of IR4.0 is limited. According to Ismail et al. (2020), students' knowledge of IR4.0 is relatively poor due to a lack of IR4.0 application in teaching and learning. Despite their lack of knowledge of IR4.0, however, the students demonstrate a strong interest in taking on the challenges it poses. This shows the students' willingness to study and develop a more nuanced knowledge of IR4.0. Thus, educational institutions must evaluate and reorganise their current curricula to create graduates who are more knowledgeable, talented, and competent in accordance with IR4.0.

Interestingly, the results of this study corroborate those of Supian et al. (2020), who discovered that students have a high degree of understanding of IR4.0. It is important to highlight, however, that a high degree of knowledge of IR4.0 does not always transfer into a high level of preparedness for IR4.0 among students. Comprehensive knowledge of IR4.0 is primarily concerned with the incorporation of IR4.0 technology into future skills and is less concerned with the students' readiness to cope with IR4.0.

As a counsellor, it is essential to possess a thorough understanding of IR4.0, especially in terms of counselling administration, which may incorporate cloud-based systems such as OneDrive and Google Drive. According to the interview data analysis, the respondents recognise the importance of IR4.0 in connection with trainee counsellor future jobs and acknowledge that IR4.0 is intrinsically connected to trainee counsellors' future professions. This is attested by three respondents in this research:

"Counsellors should be emotionally and physically prepared to deal with the technical changes that IR4.0 necessitates ..." (R1)

"Counsellors should integrate IR4.0 into their teaching and learning. Additionally, counsellors should have strong knowledge of IR4.0 to assist prospective students in navigating their potential career paths ..." (R2)

"Counsellors must possess technological knowledge in relation to IR4.0, especially in the area of counselling administration, which involves the usage of cloud system and an online counselling platform ..." (R3)

"Counsellors must develop certain skills, and new content must be added, and new programmes developed to meet the current IR4.0 demands of changes ..." (R4)

The implementation of IR4.0 has transformed the conventional administration of counselling into digital management. In terms of counselling practices, IR4.0 allows online counselling sessions to be delivered through a number of digital platforms. Online assessment for counselling is also feasible due to data analytics, which is a key pillar of IR4.0.

Employers today demand graduates to have general skills and competencies such as problem-solving, communication, interpersonal skills, lifelong learning, and the ability to work as a competent team member capable of tackling difficult issues and handling complicated circumstances on the job. As a result, educational institutions should adapt their programmes to produce graduates capable of demonstrating superior job performance and career advancement (Ahmad et al., 2019).

In terms of counselling professions, IR4.0 is inextricably linked to counsellor professionalism via the provision of digitally enhanced counselling services. According to the respondents, IR4.0 adoption is essential for future careers in this era of globalisation and digitisation. Trainee counsellors must be digitally literate in order for counsellors to stay current with technological advances. The respondents feel that it is important to include IR4.0 in the teaching and learning process when it comes to counselling professions. Additionally, the respondents suggest that counsellors should be IR4.0-literate to help students and give a thorough understanding of IR4.0 and its impact on the industry and future careers. The respondents are in favour of using virtual classrooms and tele-counselling in the implementation of IR4.0 for teaching and learning.

However, some respondents have a poor understanding of IR4.0 and are unable to make the connection between 
IR4.0 and future G\&C employment. They are unable to link the value of IR4.0 to their future job in G\&C due to their lack of understanding of IR4.0. Thus, although the trainee counsellors have a strong overall grasp of IR4.0, their knowledge of IR4.0 in relation to G\&C future careers remains modest. Corte \& Ferdinand-James (2019) proposed that, although appropriate skill development and training is not required for Industry 4.0, they are critical, with education institutions serving as an extension of industrial enterprises. Apart from that, education institutions must be supported and adopted by nearby businesses to ensure that the necessary skills are developed locally.

\subsubsection{Develop Understanding towards IR4.0 and Their Readiness}

According to the respondents, knowledge of IR4.0 is gained via both formal and informal education. Trainee counsellors are exposed to IR4.0 via specially developed technology and educational technology courses. The academic and practical elements of educational technology are covered in the courses, but according to the respondents, they have gained more knowledge about IR4.0 via informal education than institutions dedicated to increasing their knowledge and readiness for IR4.0.

The majority of trainee counsellors acquire information and comprehension of IR4.0 via news, articles, and publications, with institutions playing a less significant role in providing them with a thorough understanding of IR4.0. This idea was mentioned by all four respondents in this study:

"My knowledge of IR4.0 is derived through IR4.0-related courses and primarily from self-study ..." (R1)

"I learned about IR4.0 via my participation in extracurricular activities such as debate, forums, and conferences ..." (R2)

"My knowledge of IR4.0 is formed via both formal education such as IR4.0-related undergraduate classes and informal education such as self-reading ..." (R3)

"My knowledge of IR4.0 is mostly derived from informal education, which includes reading articles, news, and literature ..." (R4)

This study has established that trainee counsellors lack formal exposure to IR4.0. This finding is of critical importance because the lack of IR4.0 implementation by higher education institutes are shown to have a direct effect on the undergraduates' understanding of IR4.0. The current research in Malaysia has focused only on the use of technology in different courses, academic readiness, and the challenges concerned with implementing IR4.0 in education.

Sustainable development at universities is crucial because it prepares students with skills and knowledge based on what they have studied and implement it in the industry. To implement sustainable development, it is therefore essential to expand the concepts of sustainability and the importance of educational development in implementing IR4.0 (Halili et al., 2021).

Halili et al. (2021) concurred with this result and stated that the implementation of IR4.0 in higher education institutes is currently restricted and is still in its early stage. Higher education institutions should prioritise preparing students for IR4.0 in order for them to remain relevant with the demands and challenges of IR4.0. This may be accomplished by providing students with critical thinking, creativity, cooperation, and communication skills that are relevant to the implementation of IR4.0.

The primary constraint to undergraduate preparedness is a lack of digital literacy, namely the technical abilities required for IR4.0 technology implementation and digital transformation. While the respondents agree that trainee counsellors support the adoption of IR4.0, many report being unprepared to deal with it due to a lack of exposure. This is demonstrated by the four respondents:

"Despite my adequate understanding of IR4.0, I still need to prepare myself physically and psychologically to tackle IR4.0 challenges ..." (R1)

"Due to trainee counsellors' lack of exposure to IR4.0, our readiness to address the issue related to IR4.0 remains low ..." (R2)

"While training counsellors have a positive perception of IR4.0, the primary barrier is a lack of readiness and skills for IR4.0 integration ..." (R3)

"My readiness to face IR4.0 remains poor due to a lack of knowledge about IR4.0 ..." (R4)

Additionally, the respondents have voiced worries about their lack of readiness for IR4.0 and its impact on their future employment. Trainee counselors should be physically and mentally prepared to confront IR4.0. Physical readiness is intrinsically connected to technical proficiency associated with digital literacy, while mental 
readiness is linked to the capacity to learn and adapt to the changes brought about by IR4.0. Additionally, trainee counsellor's curriculum should be improved to ensure that undergraduates have a firm grasp of IR4.0, and the respondents recommend that trainee counsellors be exposed to IR4.0 to expand their knowledge and prepare them for employment in IR4.0.

The findings of this research indicate a lack of readiness among trainee counsellors to confront IR4.0, despite their high level of general awareness of IR4.0 and a reasonable degree of comprehension about how IR4.0 would be implemented in future counselling careers. However, the results of this study contradict those of Ahmad et. al. (2019), who claimed that the readiness of students in facing IR4.0 is at a high level. Undergraduates' high degree of readiness is linked to both physical and mental preparedness. As a result, students are prepared to apply the technical skills needed by IR4.0, while also being prepared to learn and adapt to the requirements of IR4.0.

Despite this, the findings from this research is confirmed by Supian et al. (2020), who concurred that undergraduates are unprepared to deal with IR4.0. Numerous variables may affect the readiness of trainee counsellors in facing the demands and challenges of IR4.0., but it is worth noting that the primary reason for the undergraduates' lack of readiness in this research is the lack of formal educational exposure to IR4.0. Thus, it is critical for education institutions to prioritise trainee counsellors in $\mathrm{G} \& \mathrm{C}$ so that they may be better prepared for future work in IR4.0 and effectively use the necessary skills. However, successful employment in IR4.0 future skills is strongly linked to the knowledge of IR4.0 and less on the readiness to confront IR4.0 (Supian et al., 2020). Thus, despite their poor readiness to face IR4.0, trainee counsellors have a high likelihood of successful employment in IR4.0 future skills owing to their high understanding of IR4.0.

\section{Conclusion}

In summary, a strong knowledge and readiness for IR4.0 is critical in preparing students for future growth in their profession. The primary goal of this study is to determine the level of knowledge and preparedness for IR4.0 among trainee counsellors at UPM. IR4.0 is powered by digital technologies and ushers in new educational paradigms by emphasising smarter, more mobile, comprehensive, and virtual education and skill development of undergraduates. The findings indicate that trainee counsellors have a solid understanding of IR4.0 in general and a moderate understanding of IR4.0 in terms of potential counselling professions. Despite this, the trainee counsellors lack readiness in facing the challenges of IR4.0, owing to insufficient formal educational exposure.

This research study is significant because it introduces new dimensions for researchers to acquire information about the comprehension and readiness of trainee counsellors for IR4.0. At the same time trainee counsellors increased self-awareness in IR4.0 future skills transformation. Additionally, this research assists education institutions in recognising the critical nature of IR4.0 adoption in teaching and learning, as well as the increased impact of IR4.0 on future skills. Thus, appropriate measures can be implemented to improve trainee counsellors' IR4.0 readiness. It is suggested that future studies include comprehensive training and incorporate additional students, employers, and quantitative analysis. While students may assert that they are unprepared for future work in IR4.0, employers may disagree. As a result, it is critical to involve employers in future study in order to get a better knowledge of this subject.

\section{References}

Abd.Kadira, R., Said, A. T., Shanmugam, S. R., Harith, H. S., \& Sidekm M. A. (2020). IR 4.0 knowledge and readiness of lecturers at the Teachers' education technical education campus, Malaysia. International Journal of Innovation, Creativity and Change, 11(11), 161-171.

Abdullah, Q., Humaidi, N., \& Shahrom, M. (2020). Industry revolution 4.0: The readiness of graduates of higher education institutions for fulfilling job demands. Romanian Journal of Infromation Technology and Automatic Control, 30(2), 15-26. https://doi.org/10.33436/v30i2y202002

Ahmad, A. R., Segaran, P., Ng, K. S., Md Sapry, H. R., \& Omar, S. S. (2019). Factors influence the students' readiness on industrial revolution 4.0. International Journal of Recent Technology and Engineering, 8(2Special Issue), 461-468.

Corte, V. D., \& Ferdinand-james, D. S. (2019). Industry 4.0: The New Industrial Revolution (pp. 138-156). https://doi.org/10.4018/978-1-5225-6207-8.ch006

Halili, S. H., Sulaiman, S., Sulaiman, H., \& Razak, R. (2021). Embracing industrial revolution 4.0 in universities. IOP Conference Series: Materials Science and Engineering, 1088(1), 012111. https://doi.org/10.1088/1757-899x/1088/1/012111 
Ismail, A., Wan Hassan. W. A. S., Ahmad, F., Affan, Z., \& Harun, M. I. (2020). Students' Readiness In Facing Industrial Revolution 4.0 Among Students Of Technical Teacher's Education. International Journal of Scientific \& Technology Research, 9, 300-305.

Jilch, S. K. (2020). Research Design and Methodology. In Cyberspace. IntechOpen. https://doi.org/10.5772/intechopen.85731

Johari, A., Abdul Wahat, N., \& Zaremohzzabieh, Z. (2021). Innovative Work Behavior among Teachers in Malaysia: The Effects of Teamwork, Principal Support, and Humor. Asian Journal of University Education, 17(2), 72-84. https://doi.org/10.24191/ajue.v17i2.13387

Lai, C. S., Chundra, U., \& Lee, M. (2020). Teaching and Learning Based on IR 4.0: Readiness of Attitude among Polytechnics Lecturers. Journal of Physics: Conference Series, 1529(3), https://doi.org/10.1088/1742-6596/1529/3/032105

Lase, D. (2019). Education and Industrial Revolution 4.0. Handayani Journal, 10(1), 48-62. https://doi.org/10.24114/jh.v10i1.14138

Manonmani, D. M., Annamalai, M. A. R., \& Veeramuthu, S. P. (2020). Literature education and industrial revolution 4.0. Universal Journal of Educational Research, 8(3), 1027-1036. https://doi.org/10.13189/ujer.2020.080337

Pu, S., Ahmad, N., Md. Khambari, M., Keng Yap, N., \& Ahrari, S. (2021). Improvement of Pre-Service Teachers' Practical Knowledge and Motivation about Artificial Intelligence through a Service-learning-based Module in Guizhou, China: A Quasi-Experimental Study. Asian Journal Of University Education, 17(3), 203-219. https://doi.org/10.24191/ajue.v17i3.14499

Sima, V., Gheorghe, I. G., Subić, J., \& Nancu, D. (2020). Influences of the industry 4.0 revolution on the human capital development and consumer behavior: A systematic review. Sustainability, 12(10), 4035 https://doi.org/10.3390/SU12104035

Supian, K., Tanius, E., \& Mohamad Idaris, R. (2020). The Malaysian Graduate Readiness to be Employed in IR 4.0. International Journal of Innovative Research in Engineering \& Multidisciplinary Physical Sciences, 8(5), 20-25. https://doi.org/10.37082/ijirmps.2020.v08i05.004

Tay, S. I., Lee, T. C., Hamid, N. Z. A., \& Ahmad, A. N. A. (2018). An overview of industry 4.0: Definition, components, and government initiatives. Journal of Advanced Research in Dynamical and Control Systems, 10(14), 1379-1387.

Zulnaidi, K., \& Majid, M. Z. A. (2020). Readiness and understanding of technical vocational education and training (TVET) lecturers in the integration of industrial revolution 4.0. International Journal of Innovation, Creativity and Change, 10(10), 31-43.

\section{Copyrights}

Copyright for this article is retained by the author(s), with first publication rights granted to the journal.

This is an open-access article distributed under the terms and conditions of the Creative Commons Attribution license (http://creativecommons.org/licenses/by/4.0/). 